\title{
Electron and Proton Radiation Effects on Band Structure and Carrier Dynamics in MBE and MOCVD Grown III-V Test Structures
}

\author{
Andrew Hudson ${ }^{1}$, Adam Scofield ${ }^{1}$, William Lotshaw ${ }^{1}$, Seth Hubbard ${ }^{2}$, Michael Slocum², Baolai Liang ${ }^{3}$, \\ Mukul C. Debnath ${ }^{3}$, Bor-Chau Juang ${ }^{4}$, Diana L. Huffaker ${ }^{4}$ \\ ${ }^{1}$ The Aerospace Corporation, 2310 E. El Segundo Blvd., El Segundo, CA, 90009 \\ ${ }^{2}$ NanoPower Research Labs, Golisano Institute for Sustainability, Rochester Institute of Technology, \\ 190 Lomb Memorial Dr, Rochester, NY 14623 \\ ${ }^{3}$ California NanoSystems Institute, 570 Westwood Plaza, Building 114, Los Angeles, CA 90095 \\ ${ }^{4}$ Electrical and Computer Engineering Department, University of California, Los Angeles, \\ 420 Westwood Plaza, Los Angeles, CA 90095
}

\begin{abstract}
As part of a study on radiation effects in optoelectronic materials, we exposed a series of AlGaAs/GaAs double heterostructures grown by molecular beam epitaxy and metalorganic chemical vapor deposition to electron and proton radiation. The active regions of the test articles were either $p$-, $n$-, or unintentionally doped. Steady state and time resolved photoluminescence spectroscopy were used to characterize radiation-induced changes to the band structure and carrier dynamics. The effect of electron radiation on low temperature photoluminescence spectra and on room temperature carrier dynamics varied with dopant type and density. Steady-state photoluminescence reveals distinct proton exposure effects for $p$ type materials grown by molecular beam epitaxy=
\end{abstract}

\section{INTRODUCTION}

On-orbit applications necessitate the investigation of radiation effects on optoelectronic devices with limited shielding from the space radiation environment. Protons can generate displacement damage and produce trap centers that degrade minority carrier lifetime [1]. While metalorganic chemical vapor deposition (MOCVD) is widely employed by commercial growers, molecular beam epitaxy (MBE) is an alternative growth technique, and the impact of radiation on test structures grown by both methods is examined.

$\boldsymbol{P}$-, $\boldsymbol{n}$-, and unintentionally doped (UID) AlGaAs/GaAs double heterostructures $(20 \mathrm{~nm}$ GaAs//100 nm $\mathrm{Al}_{0.38} \mathrm{GaAs} / / 1000 \quad \mathrm{~nm} \quad U I D / p / n-G a A s / / 100 \quad \mathrm{~nm}$ $\mathrm{Al}_{0.38} \mathrm{GaAs} / \mathrm{GaAs}$ ) were grown and spectroscopically characterized to benchmark our investigation of radiation effects as functions of doping and growth technique. An electron energy of $1 \mathrm{MeV}$ was selected due to its widespread use by the radiation effects community as well as the fact that electrons at this energy are known to generate performance degradation in GaAs solar cells. Electron fluences of $1 \times 10^{12}$, $1 \times 10^{13}, 1 \times 10^{14}$ and $5 \times 10^{14} e^{-} / \mathrm{cm}^{2}$ were selected. These values are not typical of extended missions. Fluences exceeding
$1 \times 10^{14} e^{-/ \mathrm{cm}^{2}}$ were used in a previous investigation, but induced too much material damage to permit any diagnosis of damage mechanisms via PL measurements. The proton energy $135 \mathrm{keV}$ was chosen on the basis of its performance degradation efficacy in solar cells and because protons in this energy range are known to implant in III-V materials. Proton fluences of $1 \times 10^{10}, 5 \times 10^{10}$ and $1 \times 10^{11} \mathrm{p}^{+} / \mathrm{cm}^{2}$ were employed.

\section{SAMPLE CHARACTERIZATION}

Two analytical methods were employed for benchmarking and post exposure characterization. The first is steady state photoluminesce (SSPL). A continuous wave (CW) laser excites carriers which then relax to the bandedge or to sub-bandgap defect states and radiatively recombine. The excitation laser is a Ti:Sapphire oscillator pumped with $5 \mathrm{~W} 532 \mathrm{~nm}$ and operated in CW mode. The sample PL is collected using a grating monochromator with a 600 grove/mm grating blazed at 1000 $\mathrm{nm}$. The monochromator slits are set to $30 \mu \mathrm{m}$ to achieve a resolution of $0.1 \mathrm{~nm}$ for PL spectra at $5 \mathrm{~K}$. The signal is detected by a Hamamatsu H10330B liquid nitrogen cooled photomultiplier. The $\sim 780 \mathrm{~nm}$ pump light is mechanically chopped and the detected PL signal is recovered by an SR830 lock-in amplifier. The operation of the laser in CW mode ensures that a steady state population of excess carriers is injected into the samples. The experimental system appears in Fig. 1. A fluorescent target is employed to observe Fresnel reflections off the sample surface and ensure a consistent orientation of the cryostat sample carousel, thereby maintaining a relatively constant excitation/emission geometry. The exciting laser beam was a slightly elliptical spot with axial radii of $\sim 240 \mu \mathrm{m} \times 250 \mu \mathrm{m}$.

In time resolved photo-luminescence (TRPL) a transient population of excess carriers was excited by femtosecond laser pulses generated by the Ti:Sapphire laser operating in the 
mode-locked mode with 200 fs pulse duration. The PL output is filtered by the monochromator to measure the transient PL signals at specific wavelengths, and analyzed via time correlated single photon counting (TCSPC). Figure 2 illustrates the TRPL system.

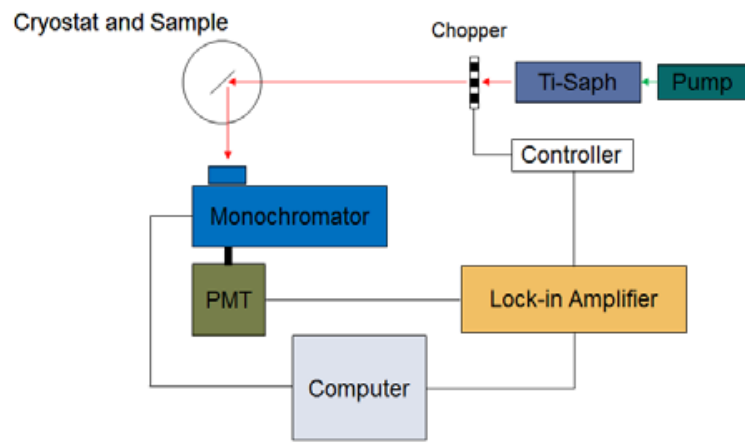

Fig. 1. SSPL characterization system.

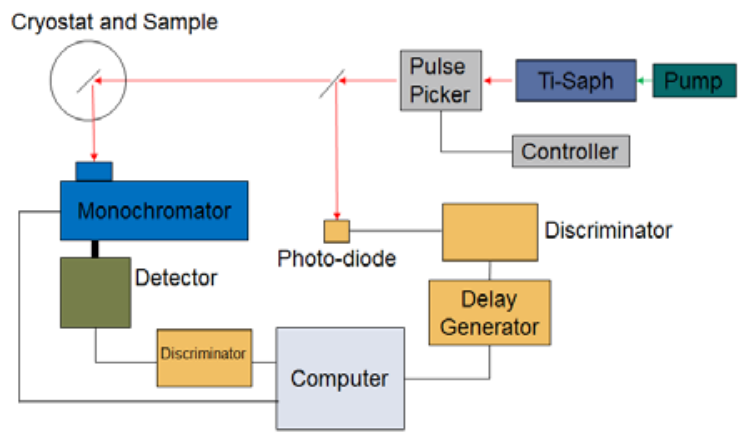

Fig. 2. TRPL characterization system.

No single decay parameter adequately describes the transient photoluminescence data, but an average lifetime can be approximated by fitting the data to a multi-exponential decay law and calculating the intensity-weighted lifetime. The fractional area of each exponential term generates the weighting factor. The intensity weighted lifetime $\tau$ is defined by

$$
\tau=\sum_{i} f_{i} \tau_{i}
$$

where $\tau_{i}$ is the $e^{-1}$ lifetime for the $i$ th fit and $f_{i}$ is the intensity weighting coefficient. These intensity weighted lifetimes are reported herein. Figure 3 shows the room temperature bandedge intensity weighted lifetimes prior to radiation exposure. Note that the lifetime for both $p$ and $n$-type specimens decreases as a function of dopant density and results from the carrier recombination which occurs at the shallow states associated with the dopants[WTL1][WTL2][AIH3].

The SSPL characterization was performed at a temperature of $5 \mathrm{~K}$ to enable the observation of luminescent recombination of bound excitons. The low temperature was achieved by mounting the samples on a rotating carousel in a closed-cycle helium cryostat. SSPL spectra were recorded prior to and following sample irradiation. TRPL characterization was performed at room temperature so that all luminescence originated at the bandedge, uncomplicated by the effects of luminescence from bound excitons.

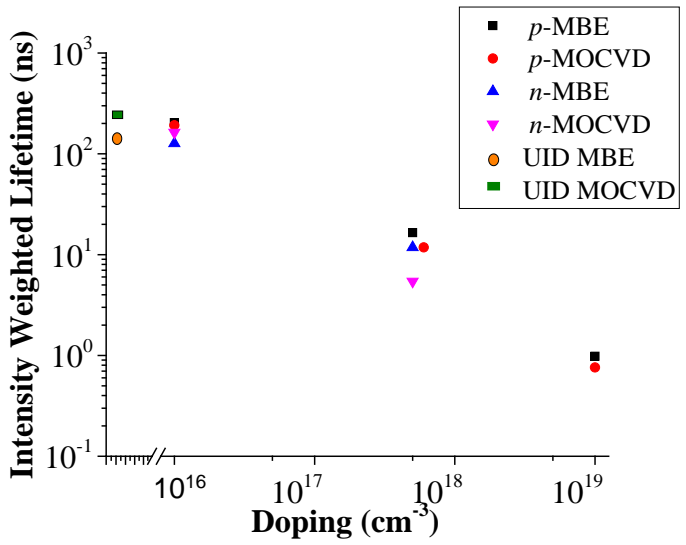

Fig. 3. Room temperature bandedge intensity weighted lifetimes prior to radiation treatment. Spot radii $\sim 240 \mu \mathrm{m} / 250 \mu \mathrm{m}$, pulse energy 1 nJ.

\section{LOW CARRIER INJECTION AND THE NON- RADIATIVE COEFFICIENT}

The relaxation of excess carriers is governed by Eq. 2 [2]:

$$
\frac{\partial \rho}{\partial t}=-\left(B N_{D}+k_{n r}\right) \rho-B \rho^{2}
$$

where $\rho$ is the carrier density, $B$ is the radiative rate coefficient, $N_{D}$ is the dopant density and $k_{n r}$ is the non-radiative rate coefficient. The first term $\left(B N_{D}\right)$ represents carrier recombination at dopant atoms, and the second term $\left(k_{n r}\right)$ the non-radiative recombination of carriers (e.g., Shockley Read Hall (SRH) recombination). Finally, the third quantity $B \rho^{2}$ represents radiative bandedge electron/hole recombination. The condition

$$
\frac{\rho}{N_{D}} \ll 1,
$$

constitutes the "low injection regime." In this regime Eq. 2 can be approximated by Eq. 4 ,

$$
\frac{\partial \rho}{\partial t} \cong-\left(B N_{D}+k_{n r}\right) \rho
$$

with an inverse of the $e^{-1}$ lifetime that is linear in the doping density,

$$
\tau_{o}^{-1}=\left(B N_{D}+k_{n r}\right)
$$

Examining the lifetime as a function of doping density facilitates an estimation of the non-radiative coefficient for each material type and radiation fluence.

The impact of radiation on a material can be quantified by the magnitude of the lifetime damage coefficient (LDC) $k_{\tau}$ [3],

$$
\tau^{-1}=\tau_{o}^{-1}+k_{\tau} \phi
$$

Here, $\tau_{0}$ is the carrier lifetime of the "as grown" material (Eq. 5), $\tau$ is the lifetime of the irradiated material, $k_{\tau}$ is the LDC and 
$\phi$ is the radiation fluence. Combining Eq. 5 and 6 gives the radiation modified non-radiative rate coefficient,

$$
k_{n r, \text { mod }}=k_{n r}+k_{\tau} \phi .
$$

Eq. 3 implies mono-exponential carrier relaxation with a lifetime described by Eq. 5. While most of the room temperature TRPL data for this study could be fitted to a single exponential, a multi-exponential decay law gave better $\chi^{2}$ fits and was therefore used in the analyses presented below. TRPL data on more complex heterostructures and at low temperature typically cannot be fitted to a first-order decay law, and require a more complex relaxation response function.

\section{ELECTRON AND PROTON RADIATION EFFECTS ON 5 K BANDSTRUCTURE}

\section{A. Electron Exposure Effects in p-Type Samples}

The $p$-type materials for both growth methods exhibit a reduction in radiative recombination as a function of increasing electron exposure. While the band structure of the samples is relatively unaffected, we observe that changes in the relative intensity of the bands appears to be mitigated by increasing the dopant density (Figs. 4 through 6). This suggests that samples with higher defect/dopant concentrations are less likely to exhibit radiation effects on their carrier recombination properties than more UID or less doped test articles.

\section{B. Electron Exposure Effects in $n$-Type Samples}

Just as for the $p$-type specimens, the $n$-type test articles from both growth methods exhibit a PL intensity which degrades as a function of increasing exposure (Figs. 7, 8). No bandstructure changes are evident but as observed in the $p$-type samples, the PL reduction appears to be mitigated by increasing dopant density, and not as pronounced as in the $p$-type samples.

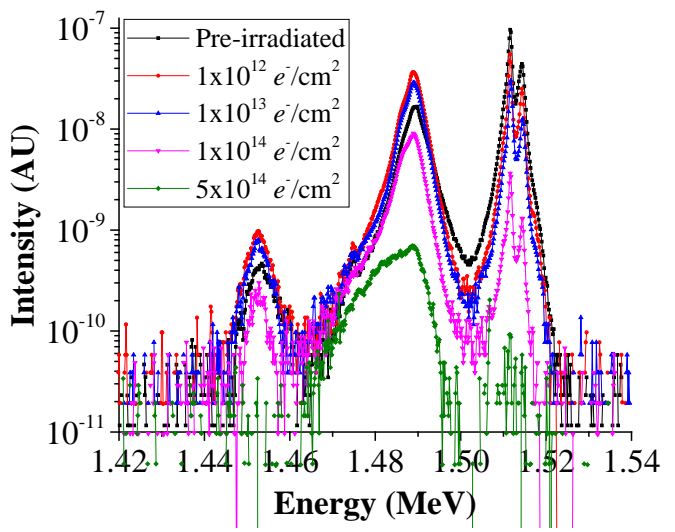

Fig. 4. Low temperature SSPL spectra for $p$-type (Zn) MOCVD grown sample prior to and after $e^{-}$exposure. Dopant density: $1 \times 10^{16} \mathrm{~cm}^{-3}$, pump power: $5 \mathrm{~mW}$; temperature: $5 \mathrm{~K}$; pump laser: $786 \mathrm{~nm}$.

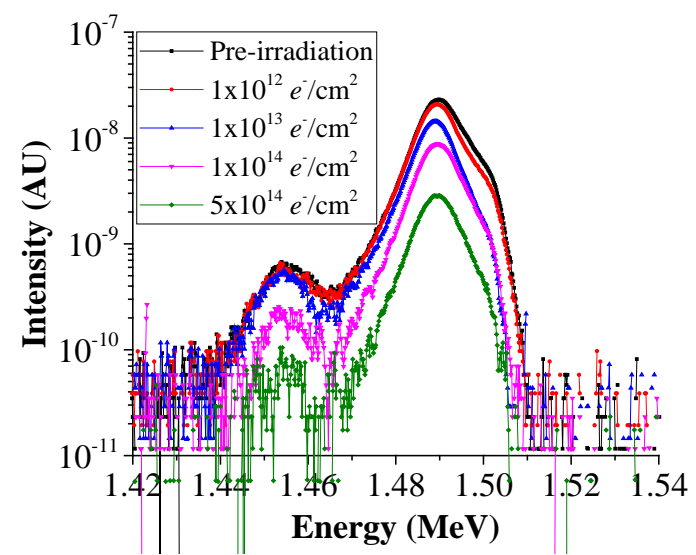

Fig. 5. Low temperature SSPL spectra for $p$-type (Zn) MOCVD grown sample prior to and after $e^{-}$exposure. Dopant density: $6 \times 10^{17} \mathrm{~cm}^{-3}$, pump power: $5 \mathrm{~mW}$; temperature: $5 \mathrm{~K}$; pump laser: $786 \mathrm{~nm}$.

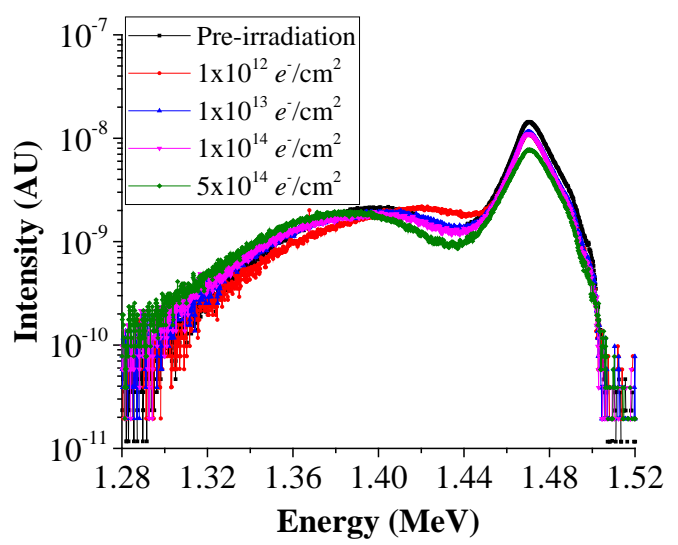

Fig. 6. Low temperature SSPL spectra for $p$-type (Zn) MOCVD grown sample prior to and after $e^{-}$exposure. Dopant density: $1 \times 10^{19} \mathrm{~cm}^{-3}$, pump power: $5 \mathrm{~mW}$; temperature: $5 \mathrm{~K}$; pump laser: $786 \mathrm{~nm}$.

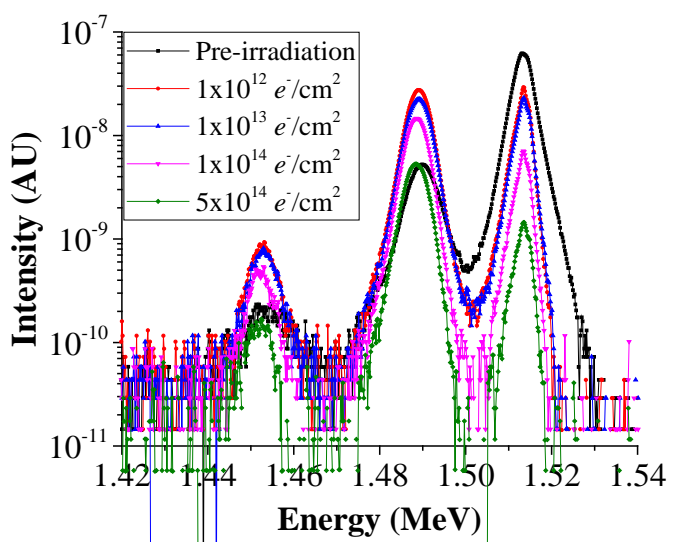

Fig.7. Low temperature SSPL spectra for $n$-type (Si) MOCVD grown sample prior to and after $e^{-}$exposure. Dopant density: $1 \times 10^{16} \mathrm{~cm}^{-3}$, pump power: $5 \mathrm{~mW}$; temperature: $5 \mathrm{~K}$; pump laser: $786 \mathrm{~nm}$. 


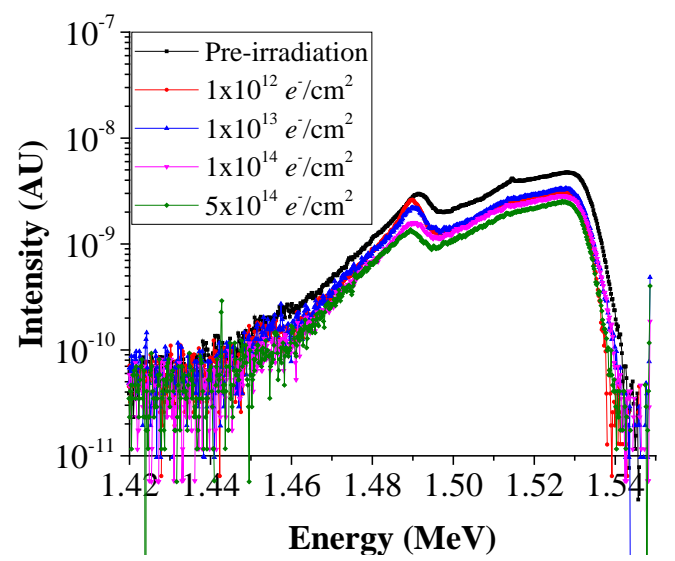

Fig. 8: Low temperature SSPL spectra for $n$-type (Si) MOCVD grown sample prior to and after $e^{-}$exposure. Dopant density: $5 \times 10^{17} \mathrm{~cm}^{-3}$, pump power: $5 \mathrm{~mW}$; temperature: $5 \mathrm{~K}$; pump laser: $786 \mathrm{~nm}$.

\section{Electron Induced Bandedge PL Reduction}

Figure 9 is a plot of the PL reduction induced by electron irradiation as a function of dopant density. The quantity examined in Fig. 9 is the ratio of the bandedge PL intensity measured after the administration of the lowest exposure and that measured after administration to the highest one:

$$
P L R F=\frac{I\left(\text { Fluence }=1 \times 10^{12} e^{-} / \mathrm{cm}^{2}\right)}{I\left(\text { Fluence }=5 \times 10^{14} e^{-} / \mathrm{cm}^{2}\right)}
$$

The $p$ - type test structure resistance to radiation induced PL degradation increases as a function of dopant density for both growth techniques, suggesting that active region doping could be adjusted for the purposes of radiation hardening. The $n-$ type test structures appear to be more inherently radiation hard than the $p$-type ones except at the highest doping density, and do not exhibit as strong a dependence on dopant density as the $p$-type samples. The impact of dopants on carrier dynamics will be considered further in the context of the room temperature lifetime damage coefficient and the non-radiative recombination coefficient.

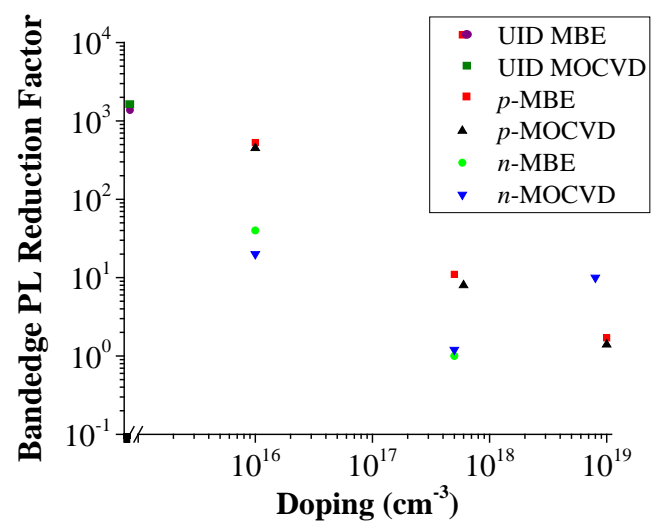

Fig. 9. $5 \mathrm{~K}$ bandedge PL reduction factor (RF). The reduction factor is the ratio of bandedge PL intensity measured after exposure to an electron fluence of $1 \times 10^{12} \mathrm{e}^{-/ \mathrm{cm}^{2}}$ to that of $5 \times 10^{14} \mathrm{e}^{-/} \mathrm{cm}^{2}$.

\section{Comparison to Proton Exposure Effects}

The radiation species used for exposure determines in part the induced bandstructure changes. The effect of $1 \mathrm{MeV}$ electron exposure does not appear to significantly alter the bandstructure (except for the relative bandedge peak amplitude at the lowest $p$ doping, see Figs 4-8), but does affect the material's carrier recombination properties. Data for the same set of test articles was collected after exposure to $135 \mathrm{keV}$ protons administered at the Aerospace Low Energy Accelerator Facility (LEAF). Figures 10 through 13 are low temperature SSPL plots for the MBE grown $p$ and $n$-type specimens doped to densities of $1 \times 10^{16} \mathrm{~cm}^{-3}$ and $5 \times 10^{17} \mathrm{~cm}^{-3}$. For the $p$ - type samples, a distinct bandstructure broadening is evident at the lowest $p^{+}$fluence with strong luminescence quenching at a higher fluence. Such broadening is not evident for the $n$-type specimens. The effects of protons on band structure can be traced to the proton energy. As mentioned above, the heterostructure exposure was simulated in the Stopping and Range of Ions in Matter (SRIM) platform with a proton energy of $135 \mathrm{keV}$. The simulations predicted that the particles would implant in the active (GaAs) region of the test articles. Implanted protons constitute trap centers in GaAs [4], which could account for the changes observed in the SSPL spectral density of the MBE $p$-type specimens. Radiation induced trap state formation in GaAs has not been as rigorously examined for $p$-type materials as for $n$-type [4], and further study is warranted. The absence of this effect in the proton exposed MBE $n$ - type samples can be explained if the majority of exciton recombination events occur at the bandedge or at shallow sub bandgap donor states. Hydrogen ions passivate shallow donor states in GaAs, possibly by the direct bonding of the hydrogen to the donor atom [5], but such passivation was not evident from the SSPL analysis.

The hypothesis described above does not explain the apparent absence of proton-induced shallow states in the SSPL spectra of the proton exposed $p$-doped (Zn) MOCVD grown test articles. This is illustrated in Fig. 14, a low temperature SSPL plots for the MOCVD grown $p$-type specimen doped to a density of $1 \times 10^{16} \mathrm{~cm}^{-3}$. It is possible that the broadening results from factors that are more complicated than proton trap state formation. It may be that the shallow states may represent complexes of the protons and native or induced defect states unique to each material.

One result which is consistent for both radiation species is the mitigating effect of dopant density for bandedge PL reduction. As illustrated in Fig. 15, the $n$-type materials proved to be more resistant to both electron and proton induced degradation effects than the $p$-type ones, and the PL intensity for both sample types at the highest fluence was increased as a function of dopant concentration. 


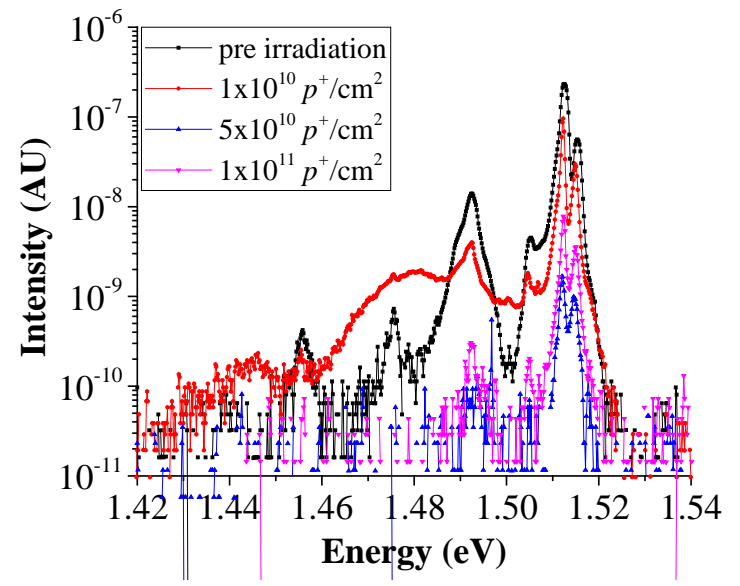

Fig. 10. Low temperature SSPL spectra for proton exposed $p$ - type (Be) MBE grown samples with dopant density $1 \times 10^{16} \mathrm{~cm}^{-3}$; pump power: $5 \mathrm{~mW}$; temperature: $5 \mathrm{~K}$; pump laser: $782 \mathrm{~nm}$.

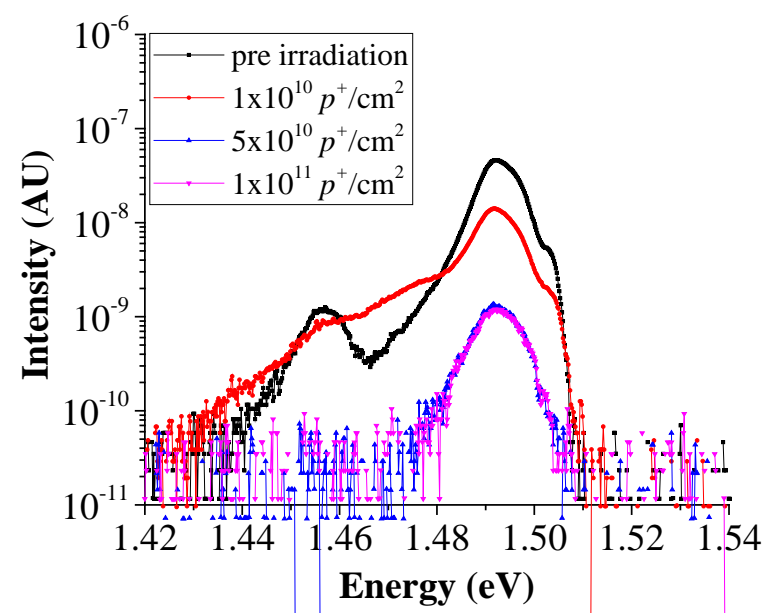

Fig. 11. Low temperature SSPL spectra for proton exposed $p$-type (Be) MBE grown samples with dopant density $5 \times 10^{17} \mathrm{~cm}^{-3}$; pump power: $5 \mathrm{~mW}$; temperature: $5 \mathrm{~K}$; pump laser: $782 \mathrm{~nm}$.

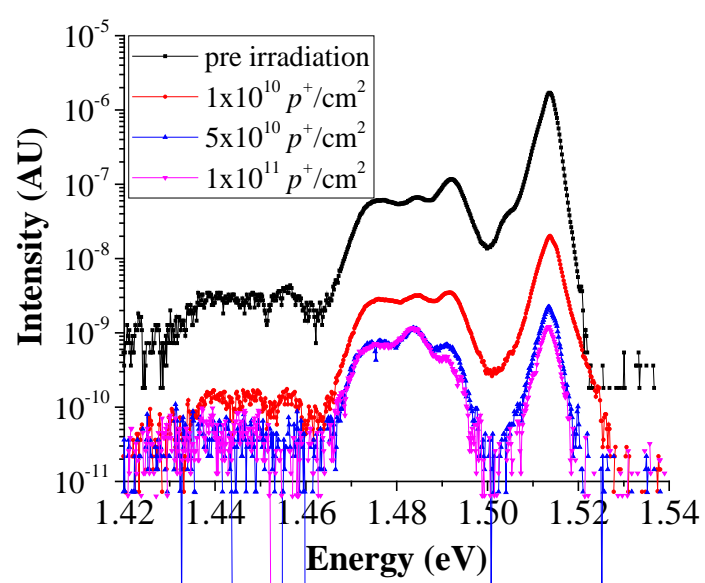

Fig. 12. Low temperature SSPL spectra for proton exposed $n$ - type (Si) MBE grown samples with dopant density $1 \times 10^{16} \mathrm{~cm}^{-3}$; pump power: $5 \mathrm{~mW}$; temperature: $5 \mathrm{~K}$; pump laser: $782 \mathrm{~nm}$.

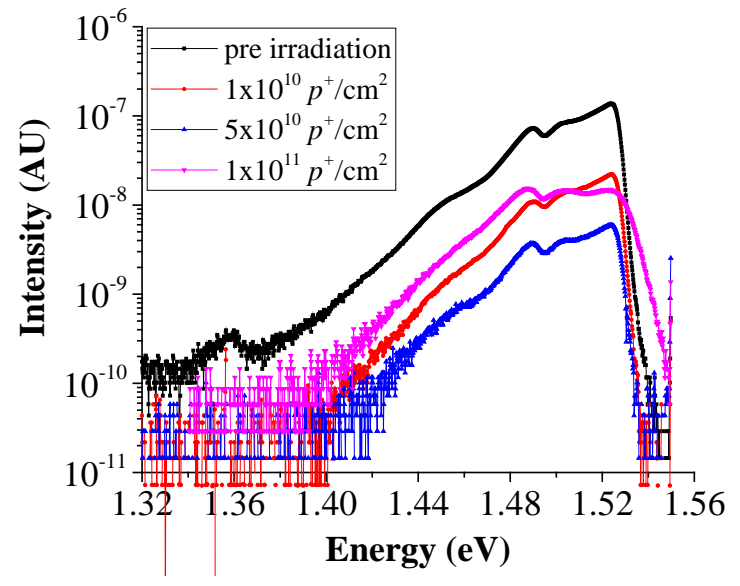

Fig. 13. Low temperature SSPL spectra for proton exposed $n$ - type (Si) MBE grown samples with dopant density $5 \times 10^{17} \mathrm{~cm}^{-3}$; pump power: $5 \mathrm{~mW}$; temperature: $5 \mathrm{~K}$; pump laser: $782 \mathrm{~nm}$.

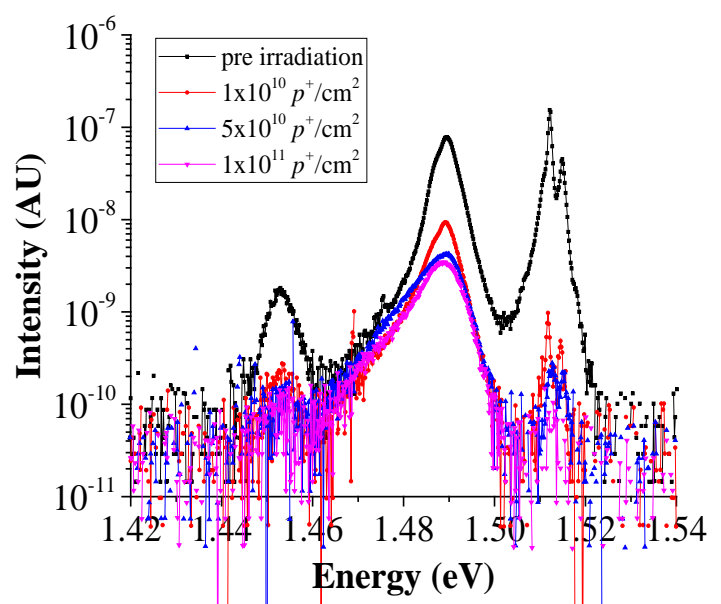

Fig. 14. Low temperature SSPL spectra for proton exposed $p$ - type (Zn) MOCVD grown samples with dopant density $1 \times 10^{16} \mathrm{~cm}^{-3}$; pump power: $5 \mathrm{~mW}$; temperature: $5 \mathrm{~K}$; pump laser: $782 \mathrm{~nm}$.

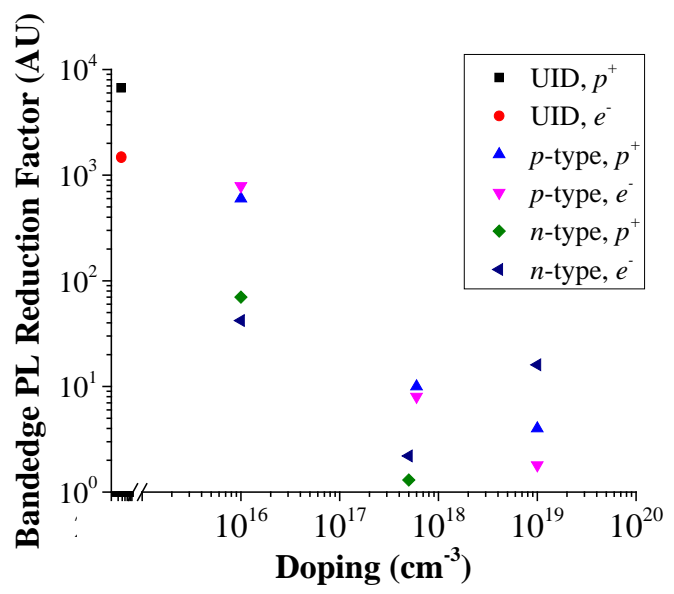

Fig. 15. $5 \mathrm{~K}$ bandedge PL reduction factor, MOCVD test articles. The reduction factor is the ratio of bandedge PL intensity measured pre exposure to that after a fluence of $5 \times 10^{14} e^{-} / \mathrm{cm}^{2}$ or $1 \times 10^{11} \mathrm{p}^{+} / \mathrm{cm}^{2}$. 


\section{ESTIMATIONS OF ROOM TEMPERATURE LIFETIME DAMAGE COEFFICIENTS AND NON-RADIATIVE COEFFECIENTS FOR ELECTRON EXPOSURE}

Room temperature TRPL data was collected at the bandedge of the test articles to determine the electron exposure LDC for each sample. The bandgaps varied from $1.425 \mathrm{eV}$ to $1.428 \mathrm{eV}$, possibly due to dopant induced bandgap renormalization [6]. TRPL lifetime data from the set of radiation fluences provides values for the LDC of each test structure (Figs. 16, 17). The LDC for the $p$-type samples at the lowest doping level is greater than that for UID test structures, but decreases as a function of increasing dopant density. This diminishing effect of radiation on carrier lifetime is consistent with the low temperature bandedge PL reduction presented in Fig. 9 and 15, except that one might expect the UID LDC to be larger than all the others due to the greater PL reduction observed at $5 \mathrm{~K}$. Figure 16 suggests that the radiation hardness of $p$ - type materials can be manipulated by adjusting the doping level. In contrast, Fig. 17 suggests that the LDC is relatively independent of doping in $n-$ type samples, paralleling the robust qualities of the $n$-type material suggested by Fig. 9 and 15 .

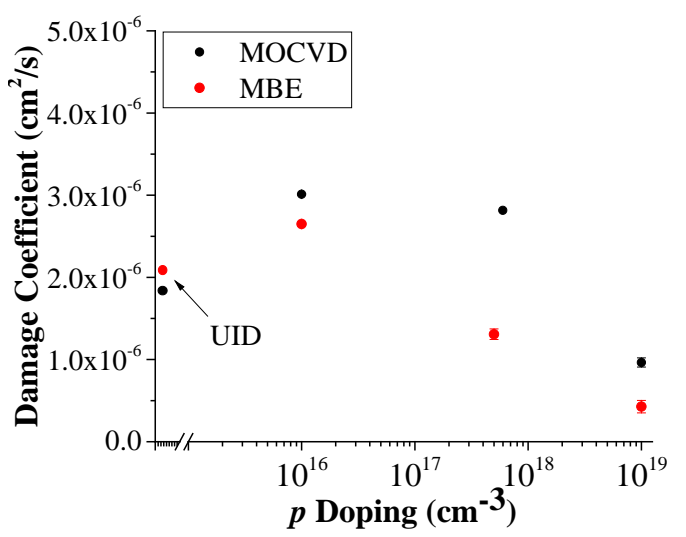

Fig. 16. Bandedge, room temperature LDC for MBE and MOCVD ptype materials.

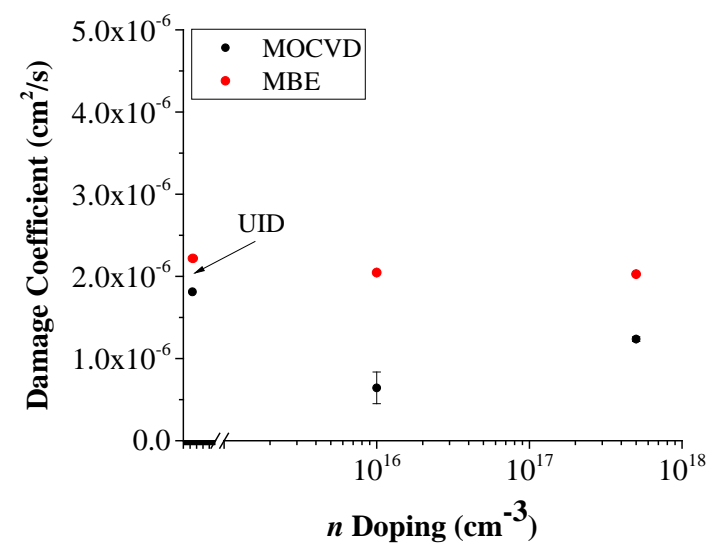

Fig. 17. Bandedge, room temperature LDC for MBE and MOCVD ntype materials.
The low injection approximation requires that Eq. 3 be satisfied, and below we estimate the injected carrier density. For this study a pulse energy of approximately $1 \mathrm{~nJ}$ was employed. The angle of incidence of the pump to the cryostat face is about $32^{\circ}$. Using Snell's law and the indices of refraction for the calcium fluoride cryostat window (1.431) as well as the GaAs cap (3.8) it was estimated that at most $14 \%$ of the pump energy excited carriers in the material. Assuming a 100\% photon to carrier conversion efficiency in the active region, an $e^{-1}$ attenuation length in GaAs of $1 \mu \mathrm{m}$ and a spot size radius of about $250 \mu \mathrm{m}$, the injected carrier density would be comparable to $1 \times 10^{15} \mathrm{~cm}^{-3}$. This is approximately an order of magnitude lower than the minimal doping level of the doped samples. The carrier density should be even less than this since reflections will occur at the AlGaAs/GaAs interface, and losses will also occur at every optic along the pump path. This carrier density estimate implies that Eq. 3 is satisfied for the data collected, and that a single exponential decay law is appropriate as suggested by Eq. 4 .

Performing a linear fit for the inverse of the bandedge carrier recombination lifetime as a function of dopant density based on Eq. 5 provides an estimate of the radiation modified nonradiative coefficient $k_{n r \text {,mod }}$ for a given dopant type, growth method and radiation fluence as the plot intercept The method assumes a constant radiative coefficient for a given material type at all doping concentrations. While this assumption may not be completely accurate, this method can provide approximate values for these quantities (Figs. 18, 19). Significant differences as a function of growth method are not evident, and all increase almost linearly with radiation fluence as predicted by Eq. 7. One point to note is that there are no uncertainty estimates for the $n$-type samples: TRPL data was not collected for the highest doping concentration due to the fact that no resolvable bandstructure was observed at room temperature. Only two data points could be obtained for the $n$ type $k_{n r, \bmod }$ estimates. The linearity as a function of fluence facilitates an estimation of the change in the non-radiative coefficient per unit fluence derived from fits to the data in Figs. 18 and 19. This quantity, comparable to the LDC according to Eq. 7, appears in Fig. 20. The values are distinct to 3- $\sigma$ and those for the $n$-type materials are smaller than those for the $p$ type. These results again suggest that the $n$-type specimens are more radiation hard to $1 \mathrm{MeV}$ electron effects than the $p$-type samples.

The data fit intercepts for Figs. 18 and 19, representing the zero exposure, non-radiative coefficient $k_{n r}$ for each growth method and doping type according to Eq. 7 appear in Fig. 21. The value for the $n$-type MOCVD grown specimen is distinct from the rest to $1-\sigma$ but not $2-\sigma$. This suggests that at most there is only little variancebetween the intrinsic non-radiative recombination properties of the materials, and that the defects generated in each type of sample during exposure cause unique changes to $k_{n r}$. 


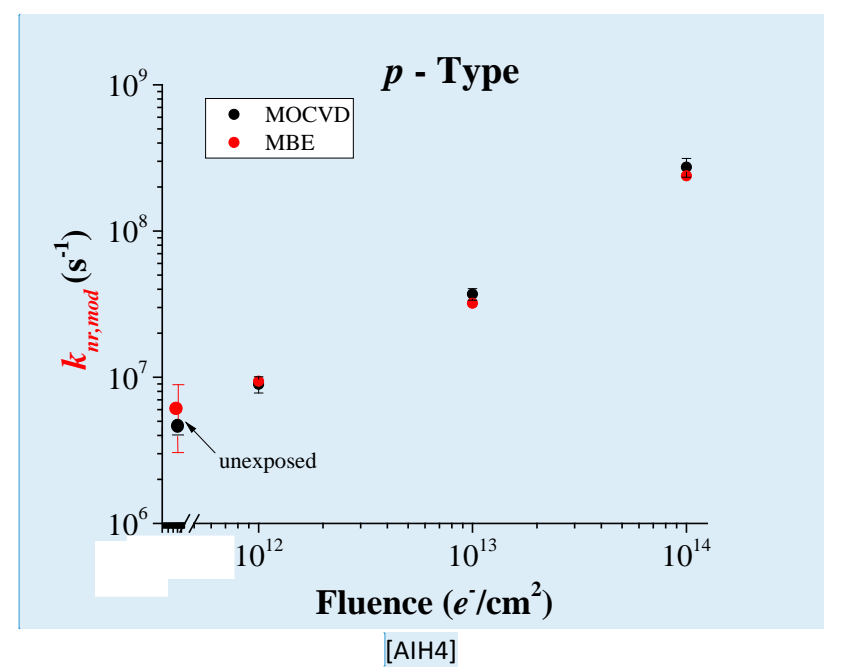

Fig. 18. Bandedge, room temperature non-radiative rate coefficients for MBE and MOCVD $p$-type materials.

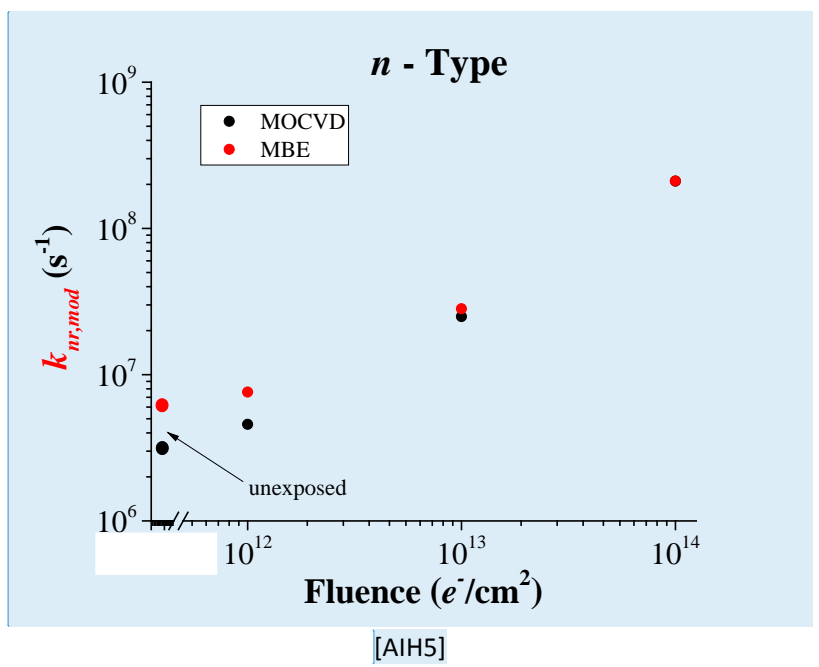

Fig. 19. Bandedge, room temperature non-radiative rate coefficients for MBE and MOCVD $n$-type materials.

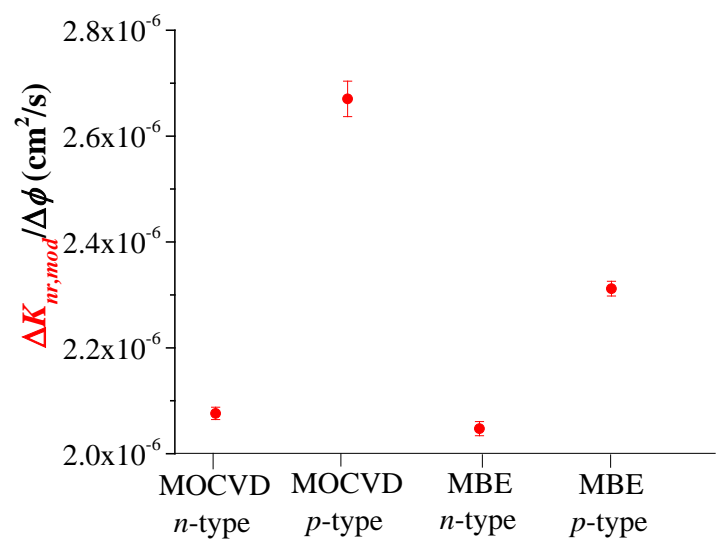

Fig. 20. Change in the room temperature non-radiative rate coefficient per unit fluence for the $n$ and $p$ - type materials.

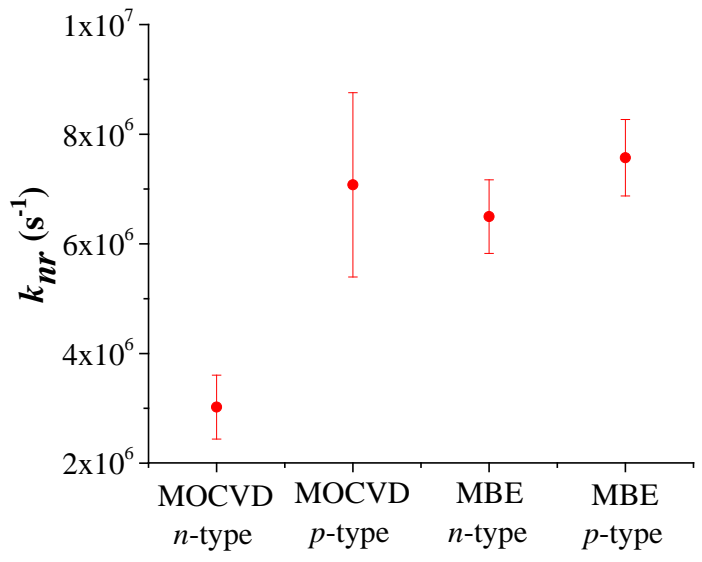

Fig. 21. Residual room temperature non-radiative rate coefficient for the $n$ and $p$ - type materials.

An analysis comparable to the one above for electron exposure could not be performed for the proton irradiated specimens. The lifetime fits for proton irradiated samples were poor in comparison to those electron exposures, with low $R^{2}$ coefficients as represented in Fig. 22 and 23. This might result from the sub $\mathrm{MeV}$ particle energy. An energy of $135 \mathrm{keV}$ was selected to implant the protons into the active region material, but this will also generate a spatial dependence in the induced defect distribution, with the greatest amount of displacement damage occurring at the termination of the particle track [7]. Eq. 2 assumes a constant defect density and cannot be applied to materials with isotropic ones. An MeV energy selection such as customarily used for the qualification of solar cells and detector devices would have facilitated a near constant linear energy transfer in the material, and hence a constant defect density throughout it.

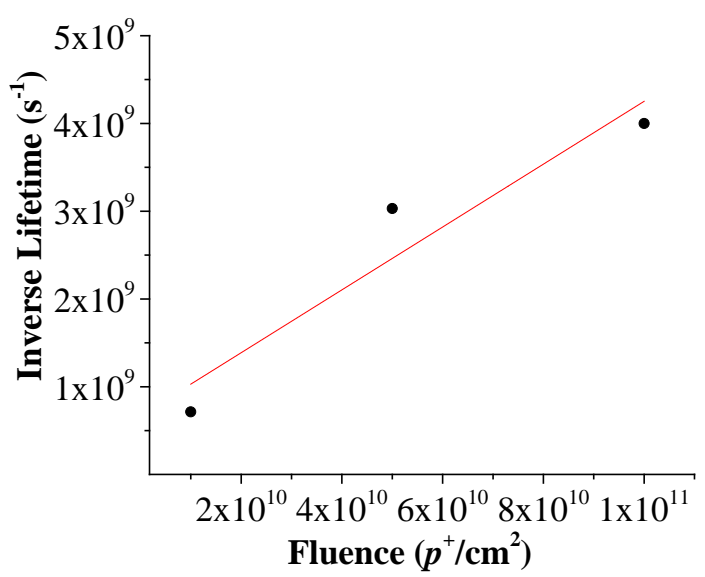

Fig. 22. Bandedge, room temperature inverse lifetime plot for proton exposed, $p$-type MOCVD grown sample with a dopant (Zn) density of $1 \times 10^{16} \mathrm{~cm}^{-3}$. Adjusted $R^{2}$ coefficient: 0.83 . 


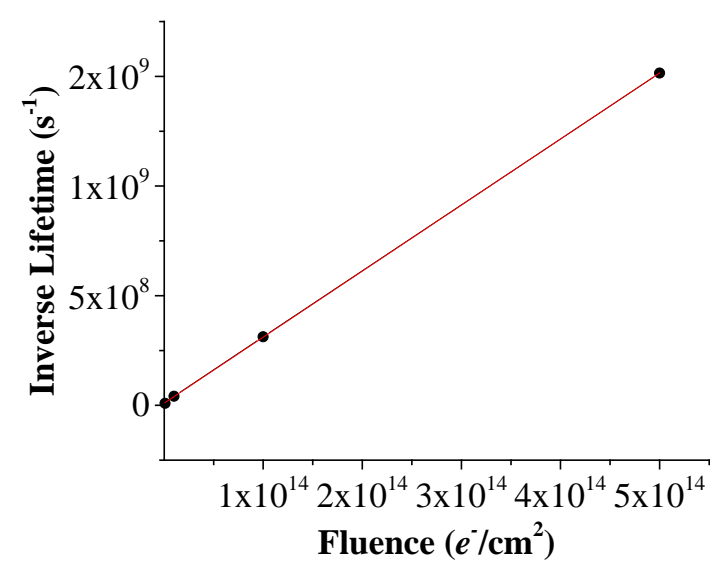

Fig. 23. Bandedge, room temperature inverse lifetime plot for electron exposed, $p$-type MOCVD grown sample with a dopant (Zn) density of $1 \times 10^{16} \mathrm{~cm}^{-3}$. Adjusted R $\mathrm{R}^{2}$ coefficient: 1.00 .

\section{ANALYSIS AND FINDINGS}

The effects of proton and electron exposure were substantial and distinct. While SSPL is not as comprehensive a defect probe as Deep Level Transient Spectroscopy (DLTS), it does enable identification of luminescent trap states [8]. The idea that additional states resulting from proton implantation in $p$ type test articles appear to form a continuous distribution above the valence band explains the results of the MBE sample exposures but not the MOCVD result, which could be a consequence of the formation of different complexes associated with each dopant atom (Be or Zn). The absence of this effect for the $n$ - type specimens for both growth methods can be understood if the majority of exciton recombinations in these samples occurs at the bandedge or through shallow donor states.

The doping dependence of the low temperature bandedge PL reduction for both $p^{+}$and $e^{-}$radiation species suggests that the $p$-type samples were more susceptible to radiation induced nonradiative recombination mechanisms than the $n$-type ones, and that this effect is mitigated by increased doping. The doping dependence of the room temperature LDC for the electron exposed specimens supports this interpretation. Also, the nonradiative rate coefficients for the $n$-type samples did not increase as much per unit fluence in comparison to the $p$-type ones. The cumulative evidence indicates that the $n$-type specimens are more radiation hard than the $p$-type ones, and less affected by either $1 \mathrm{MeV}$ electron or $135 \mathrm{keV}$ proton exposure. Determining the changes in material properties as a function of radiation species, dopant type and dopant density facilitates an understanding of the basic physics of performance degradation and has the potential to identify the regions of photovoltaic devices particularly prone to orbit-specific radiation induced material breakdown.

\section{SUMMARY}

Electron exposure did not generate the spectroscopically resolvable trap states observed with proton exposure, but did alter the carrier recombination properties of the materials. Electron-induced changes in PL intensity and carrier lifetime were similar for MBE and MOCVD grown materials. $n$-type samples appeared to be more radiation hard compared to $p$-type ones. The emerging results of our ongoing study targets the material physics basis for the proposition that dopant type and density may be viable optimization parameters for mitigation of on-orbit degradations of optoelectronic device performance caused by radiation exposure (e.g., solar cells and focal plane arrays).

On the basis of our results with simple GaAs/AlGaAs heterostructures, we are expanding the scope of these studies to include emerging III-V material structures for next-generation IR detectors such as InAs/InAsSb superlattices to assess their susceptibility to radiation exposure.

\section{ACKNOWLEDGEMENTS}

The authors acknowledge Dr. Brandon Wall of the Boeing Radiation Effects Laboratory for performing the electron exposures, and Mr. Colin Mann and The Energy Technology Department at The Aerospace Corporation for executing the proton exposures.

\section{REFERENCES}

1. N. Chandrasekaran, T. Soga, Y. Inuzuka, H. Taguchi, M. Imaizumi, T. Oshima, T. Jimbo, "Low-energy proton irradiation effects on GaAs/Si solar cell”, Japanese Journal of Applied Physics,Vol.43, No. 10A, pp. L1302-L1304, 2004.

2. R K. Ahrenkiel, M.S. Lundstrom, "Minority carriers in III Vsemiconductors, physics and applications, semiconductors and semimetals”, Vol. 39, New York, New York: Academic Press, 1993.

3. S. M. Khanna A. Houdayer, A. Jorio, C. Carlone, M. Parenteau, J.W. Gerdes Jr., "Nuclear radiation displacement damage prediction in gallium arsenide through low temperature photoluminescnece measurements", IEEE Transactions on Nuclear Science, Vol. 43, No. 6, pp. 2601-2608, 1996.

4. S.J.Pearton W.C. Dautremont-Smith, J. Chevallier, C.W Tu,K.D.Cummings, "Hyrogenation of shallow-donor levels in GaAs”, Journal of Applied Physics, Vol. 59, pp. 2821-2827, 1986.

5. M. Gonzalez, C.L Andre, R.J. Walters, S.R Messinger, J.H. Warner, J.R. Lorentzen, A.J. Pitera, E.A.Fitzgerald, S.A. Ringel, "Deep level defects in proton radiated GaAs grown on metamorphic SiGe/Si substrates", Journal of Applied Physics, Vol. 100, 034503, pp. 1-7, 2006.

6. P. Y. Yu, M. Cardona, "Fundamentals of semiconductors, physics and material properties”, $4^{\text {th }}$ Ed., pp. 342-355, Springer - Verlag, 2010.

7. B. E. Anspaugh, "GaAs solar cell radiation handbook," JPL Publication 96-9, pp. 4-12, 1996.

8. P.K. Bhattacharya and S.J.T Owen, "New radiative deep states in epitaxial Ga1-xAlxAs”, Applied Physics Letters, Vol. 36, No.8, pp. 664-666, 1980. 